\title{
INFLUENCIA DEL PH EN LA LIBERACIÓN DEL FACTOR DE CRECIMIENTO DERIVADO DE PLAQUETAS (PDGF-BB) A PARTIR DE UN HIDROGEL A BASE DE B-QUITOSANO
}

\author{
Sthefanie Cabrel Rengifo ${ }^{1 *}$, Graciela Untiveros Bermúdez², José Luis Aguilar Olano ${ }^{3}$
}

\begin{abstract}
RESUMEN
Se desarrolló un hidrogel con matriz de $\beta$-quitosano que retarde la liberación del factor de crecimiento derivado de plaquetas - BB (PDGF - BB), el cual forma parte del lisado plaquetario (LP), obtenido a partir del plasma rico en plaquetas (PRP). Para la elaboración del hidrogel se preparó la solución de $\beta$-quitosano 1,5\% (p/v) en ácido acético $2 \%(\mathrm{v} / \mathrm{v}), \mathrm{LP}$ al $6 \%$ o $12 \%$ (v/v) y glutaraldehído a una concentración final de $0,03 \mathrm{M}$. El $\beta$-quitosano usado como materia prima, se caracterizó por tener $12,47 \%$ de humedad relativa promedio, grado de desacetilación promedio de 94,53 \% y peso molecular promedio de 145,94 kDa; atributos importantes que permitieron formular un hidrogel de consistencia semi-sólida, incoloro y fácil de manipular. La liberación retardada se logró con el hidrogel con $12 \%$ de LP, pues la liberación de PDGF - BB no difiere significativamente $(\mathrm{p}<0,05)$ al variar el $\mathrm{pH}$ de 6,5 $(5$ 491,42 pg de PDGF - BB/mL de LP) a 7,4 (5 387,39 pg de PDGF - BB/mL de LP) luego de 32 horas. Por el contrario, el hidrogel con $6 \%$ de LP, sí mostró una diferencia significativa en la cantidad de PDGF - BB liberado al variar el pH de 6,5 (12 687,66 pg de PDGF - BB/mL de LP) a 7,4 (9 396,58 pg de PDGF - BB/mL de LP) luego de 32 horas.
\end{abstract}

Palabras clave: $\beta$-quitosano, hidrogel, liberación retardada, PDGF-BB.

\section{INFLUENCE OF PH ON RELEASING PLATELET-DERIVED GROWTH FACTOR (PDGF-BB) FROM A B-CHITOSAN-BASED HYDROGEL}

\begin{abstract}
A hydrogel with $\beta$-chitosan matrix that retards the release of platelet-derived growth factor $\mathrm{BB}$ (PDGF - BB), which is part of the platelet lysate (LP) obtained from platelet rich plasma (PRP), was developed. The hydrogel was composed of a solution of $1.5 \%(\mathrm{w} / \mathrm{v}) \beta$-chitosan in $2 \%(\mathrm{v} / \mathrm{v})$ acetic acid, $6 \%$ or $12 \% \mathrm{LP}(\mathrm{v} / \mathrm{v})$ and glutaraldehyde $0.03 \mathrm{M}$. The $\beta$-chitosan used as raw material had $12.47 \%$ of average relative humidity, 94.53 of average deacetylation

${ }^{1}$ Universidad Peruana Cayetano Heredia, Lima - Perú, sthefanie.cr@gmail.com

${ }^{2}$ Sección de Química. Facultad de Ciencias. Universidad Peruana Cayetano Heredia, Lima - Perú.

${ }^{3}$ Laboratorio de Inmunología. Departamento de Ciencias Celulares y Moleculares

Facultad de Ciencias. Universidad Peruana Cayetano Heredia, Lima - Perú.
\end{abstract}


degree and an estimated average molecular weight of $145.94 \mathrm{kDa}$; important attributes to formulate a hydrogel of semi-solid consistency, colorless and easy to handle. Delayed release was achieved with the $12 \%$ LP hydrogel since the release of PDGF - BB does not differ significantly $(\mathrm{p}<0.05)$ by varying the $\mathrm{pH}$ from 6.5 (5 $491.42 \mathrm{pg}$ of $\mathrm{PDGF}-\mathrm{BB} / \mathrm{mL}$ of LP) to 7.4 (5 $387.39 \mathrm{pg}$ of PDGF - BB/mL of LP) after 32 hours. In contrast, the $6 \%$ LP hydrogel show a significant difference in the amount of PDGF-BB released by varying the $\mathrm{pH}$ from 6.5 (12 687.66 pg of PDGF - BB/mL of LP) to 7.4 (9 $396.58 \mathrm{pg}$ of PDGF - BB/mL of LP) after 32 hours.

Key words: $\beta$-chitosan, hydrogel, delayed release, PDGF-BB.

\section{INTRODUCCIÓN}

Las úlceras cutáneas son problemas de salud frecuentes cuya cronicidad es el principal detonante de complicaciones, como las infecciones. Por la composición del estrato córneo, el $\mathrm{pH}$ superficial basal de la piel está cerca de $5^{1}$. Sin embargo, ante un daño tisular (o herida) la concentración efectiva de iones hidronio en el medio varía hasta terminar la fase de granulación o proliferación, donde el $\mathrm{pH}$ se encuentra alrededor de 7,52. Si la herida llega a esta fase, se la clasifica como aguda o fisiológica; por otro lado, si continúa desordenadamente entre la fase inflamatoria y la proliferativa por más de tres semanas, se la considera crónica o patológica1. Este tipo de herida no logra normalizar los valores de $\mathrm{pH}$ ácidos debido a las deficiencias en el mecanismo reparador; por lo que se mantiene en medio alcalino, propenso al crecimiento bacteriano ${ }^{1,2}$.

Ante la problemática, diversas terapias que se asemejan al mecanismo reparador natural son estudiadas, como lo es el uso de factores de crecimiento. El factor de crecimiento derivado de plaquetas-BB (PDGF-BB) es una glicoproteína catiónica con punto isoeléctrico a pH 9,8; que ha demostrado efectividad en acelerar el proceso de cicatrización in vitro con el aumento en la proliferación de fibroblastos ${ }^{3,4}$. Sin embargo, los efectos terapéuticos de su aplicación en monoterapia no se comparan con el sinergismo junto a otros factores de crecimiento 4 . Además, uno de los principales inconvenientes del uso de este tratamiento en úlceras cutáneas crónicas, es la inactivación de los factores de crecimiento por altos niveles de metaloproteasas. Es por ello que una liberación funcional con la adecuada matriz que no solo sea compatible, sino que promueva la actividad de los factores de crecimiento, es crucial ${ }^{5}$.

Este trabajo propone un hidrogel como forma farmacéutica que pueda retardar la liberación del PDGF-BB usando una matriz de $\beta$-quitosano. La estructura tridimensional estará formada por una red de polímeros entrecruzados con un alto número de grupos o dominios hidrofílicos que, al atraer agua, se "hinchan" hasta llegar a un equilibrio entre la fuerza que ejercen las moléculas del medio al entrar a la matriz (fuerza expansiva) y la fuerza de atracción entre las cadenas del polímero (fuerza retráctil) ${ }^{6}$. El $\beta$-quitosano es un polímero con muchas propiedades dependiendo del grado de desacetilación y el peso molecular promedio: antibacteriano, antifúngico, mucoadhesivo, analgésico, hemostático, biodegradable, 
biocompatible y promotor de la proliferación celular mediada por factores de crecimiento $^{5}$. Estas redes de quitosano pueden seguir tres mecanismos de encapsulación dependiendo de la IFA cargada: difusión, atrapamiento o unión covalente ${ }^{6}$. Los enlaces covalentes son las uniones por excelencia en la formación de la red, aunque no son las únicas. Los puentes de hidrógeno y las interacciones hidrofóbicas juegan un rol importante en la formación de un hidrogel de interacciones físicas?

El objetivo del trabajo es estudiar la influencia del $\mathrm{pH}(6,5$ y 7,4) en la liberación de uno de estos factores de crecimiento (PDGF-BB) a partir de una matriz de $\beta$-quitosano bajo dos concentraciones de lisado plaquetaria o LP (6 \% y $12 \%)$. Con ello, se sabrá si el diseño de la forma farmacéutica es teóricamente efectivo en el tratamiento de úlceras cutánea. Se espera que la formulación contenga suficientes uniones covalentes y/o iónicas entre la proteína y el polímero para que, al exponerse a variaciones en el $\mathrm{pH}$ del medio, como ocurre en las úlceras cutáneas, retarde la liberación de PDGF-BB.

\section{PARTE EXPERIMENTAL}

El polímero de $\beta$-quitosano fue provisto por el Laboratorio de la Unidad de Biominería y Medio Ambiente de la Universidad Peruana Cayetano Heredia. La muestra de PRP se obtuvo de un individuo sano sometido a plasmaféresis posterior al consentimiento informado, en cumplimiento a las buenas prácticas clínicas a cargo del Laboratorio de Inmunología de la Universidad Peruana Cayetano Heredia. Para la cuantificación de PDGF-BB humano se usó el Kit DuoSet ELISA Development Systems: R\&D Systems (Número de catálogo: DY220).

\section{Caracterización del $\beta$-Quitosano}

La materia prima fue triturada con un molino analítico modelo $4301-02,50 / 60 \mathrm{~Hz}, 180 \mathrm{~W}$, 20000 RPM, marca Cole-Parmer Instrument Co. y tamizada por malla de acero W.S. TYLER $\mathrm{n}^{\circ} 60$, para tener un tamaño de partícula homogéneo. El \% humedad relativa se determinó por gravimetría, llevando a peso constante (diferencia de $0,05 \mathrm{~g}$ ) una cantidad de muestra expuesta a $105^{\circ} \mathrm{C}^{(8)}$.

El grado de desacetilación (GD) se determinó mediante una valoración potenciométrica. Se disolvió $\beta$-quitosano en un exceso conocido de $\mathrm{HCl} 0,2 \mathrm{~N}$; la misma que fue titulada con una solución de $\mathrm{NaOH} 0,05 \mathrm{~N}$. Las medidas de $\mathrm{pH}$ fueron graficadas identificando dos puntos de inflexión; la diferencia en mililitros entre ambos puntos corresponde al ácido consumido para la protonación de grupos amino, los que permiten determinar el GD del quitosano ${ }^{8}$.

La siguiente ecuación se usó para calcular el GD:

$$
\mathrm{GD}=100-\frac{100 \times \text { Normalidad }_{\mathrm{NaOH}} \times \mathrm{V} \times \mathrm{PEq}_{\mathrm{NH}_{2}}}{\text { Peso de la muestra }}
$$

Donde: $\mathrm{V}$ es la diferencia de volúmenes (litros) entre el segundo y primer punto de inflexión y PEq (NH2) es 16,02258 g/Eq. 
Para hallar el peso molecular promedio se aplicó su relación a la viscosidad intrínseca. Para ello se usó un viscosímetro de Ostwald con diámetro $0,5 \mathrm{~mm}$ y a condiciones ambientales controladas (25 $\left.{ }^{\circ} \mathrm{C} \pm 0,1\right)$. El solvente usado fue Ácido acético $0,3 \mathrm{M} /$ Acetato de Sodio $0,2 \mathrm{M}^{8,9}$. La ecuación usada fue la de Mark-Houwink-Sakurada:

$$
[\eta]=\mathrm{KM}_{\mathrm{v}}{ }^{\mathrm{a}}
$$

Donde [ $\eta$ ] es la viscosidad intrínseca, $\mathrm{Mv}$ es el peso molecular promedio, $\mathrm{K}$ y $\alpha$ son constantes dadas para un sistema soluto-solvente. Para la ecuación se consideraron los valores de 0,076 mLg-1 y 0,76 para $\alpha$ y K, respectivamente; en función del GD, origen del quitosano y solvente usado ${ }^{10}$.

\section{Elaboración del hidrogel, incubación y cuantificación de PDGF-BB}

El experimento sigue un diseño factorial fraccionado de dos niveles: \%LP y pH del medio de liberación.

El LP derivó del PRP obtenido tras someter a un donante sano a una plasmaféresis por centrifugación de flujo discontinuo. Este procedimiento permite la separación del plasma de los otros componentes sanguíneos, logrando obtener al finalizar un pool de plasma puro y rico en plaquetas, que para este trabajo tuvo una concentración de $1,5 \times 10^{6}$ plaquetas.

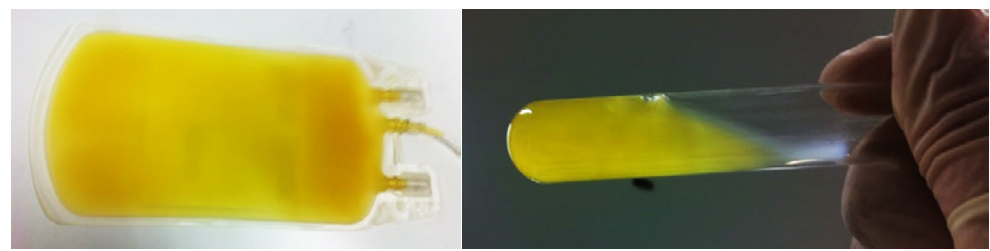

Figura 1. Plasma recolectado tras plasmaféresis por centrifugación de flujo discontinuo.

Para obtener el LP, se tomó $10 \mathrm{~mL}$ del PRP y sometió a un shock de temperatura para lisar de plaquetas. Para esto, se congeló a $-70^{\circ} \mathrm{C}$ por 12 horas y descongeló a $37^{\circ} \mathrm{C}$ (aproximadamente 10 minutos), lo que se denomina un ciclo. Después de tres ciclos, se centrifugó la muestra a 2500 rpm durante 15 minutos; el sobrenadante es el LP ${ }^{11}$.

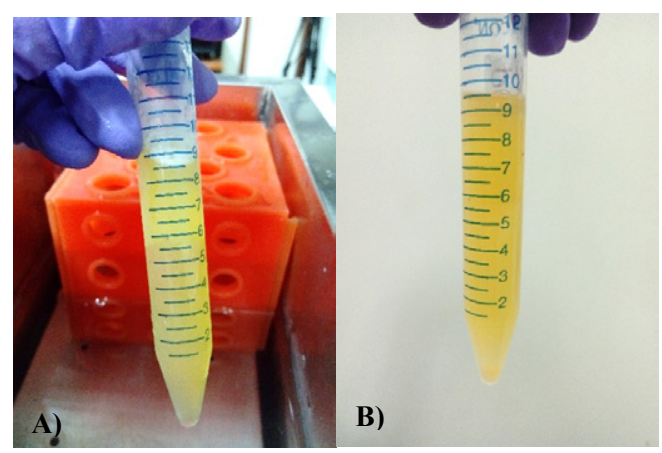

Figura 2. A) PRP en descongelamiento B) PRP post centrifugación. 
Para la elaboración del hidrogel, se preparó la solución de $\beta$-quitosano 1,5\% (p/v) en ácido acético $2 \%(\mathrm{v} / \mathrm{v})^{12,13}$, luego se adicionó LP al $6 \%$ o $12 \%(\mathrm{v} / \mathrm{v})$ hasta homogeneizar con ayuda de un agitador magnético. Por último, se adicionó glutaraldehído al $25 \%$ con una concentración final de $0,03 \mathrm{M}^{12}$ e inmediatamente agitar vigorosamente. Sin dejar reposar más de un 1 minuto, se traspasó $1 \mathrm{ml}$ de la mezcla en cada pozo de la placa de 24 pozos y se dejó reposar entre 45 a 60 minutos.

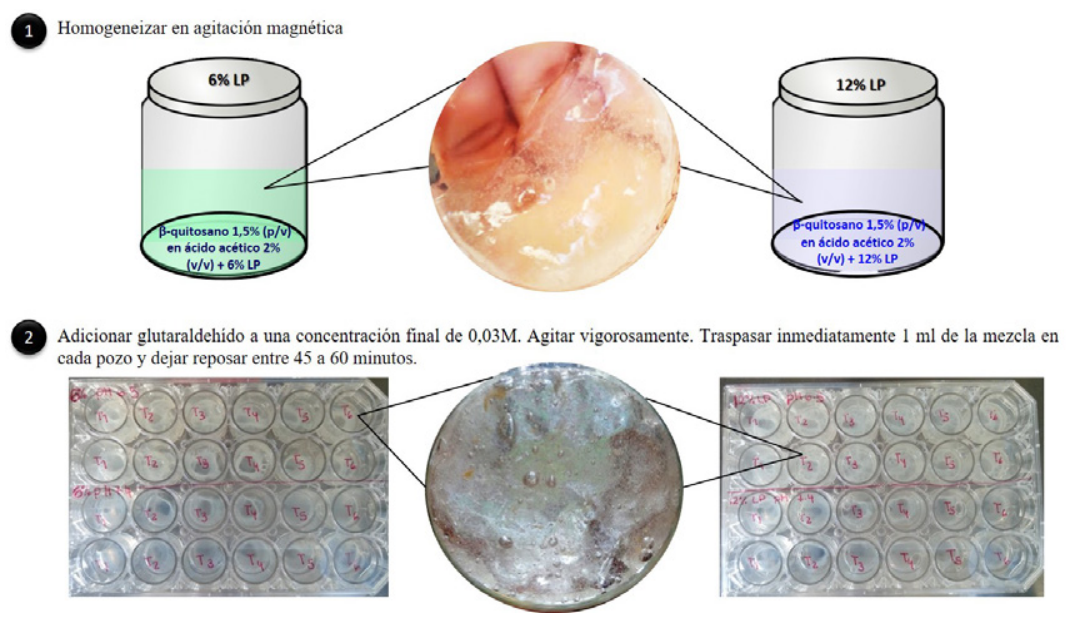

Figura 3. Formación del hidrogel.

En referencia al pH del medio, a cada pozo con el hidrogel de la placa se agregó $400 \mu \mathrm{L}$ del PBS a un pH determinado $(6,5$ o 7,4). Es importante cumplir con el tiempo de reposo para la completa formación del hidrogel antes de colocar el PBS; de lo contrario, la consistencia del mismo puede destruirse.

Una vez colocado el PBS, la placa permaneció en la incubadora $\left(5 \% \mathrm{CO}_{2}\right)$ hasta llegar el tiempo para las cuantificaciones de PDGF-BB, las cuales se realizaron por triplicado de cada pozo, bajo las especificaciones del producto DuoSet ${ }^{\circledR}$ ELISA para PDGF-BB (Número de Catálogo: DY220).

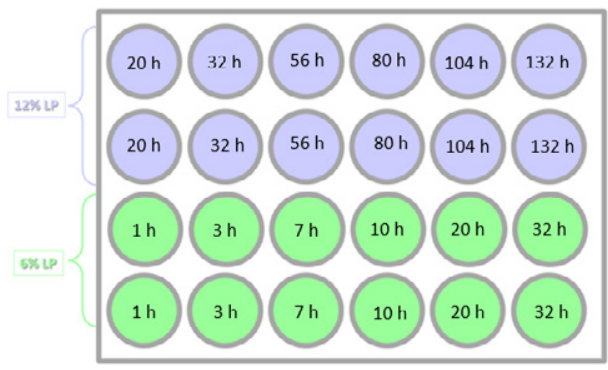

Figura 4. Esquema de distribución de la placa para el experimento según el tiempo para las cuantificaciones de PDGF-BB. 


\section{Pruebas estadísticas para el contraste de hipótesis}

Se comparó la concentración de PDGF-BB como variable numérica en dos grupos no relacionados en base al porcentaje de lisado plaquetario. Los datos en ambos grupos fueron sometidos a pruebas de normalidad, linealidad y homogeneidad de varianzas, habiéndose decidido usar la prueba no paramétrica de Kruskal-Wallis.

\section{RESULTADOS Y DISCUSIÓN}

\section{Caracterización del $\beta$-Quitosano:}

El $\beta$-Quitosano usado como materia prima se caracterizó por un tamaño de partícula de $250 \mu \mathrm{m}$ como máximo y un porcentaje de humedad relativa promedio de $12,47 \pm 0,24$. El grado de desacetilación promedio (GD) expresado en porcentaje, una de sus principales características, fue de $94,53 \pm 0,84$. Mientras que el peso molecular promedio estimado fue de $145,94 \mathrm{kDa}\left(\mathrm{R}^{2}=0,9876\right)$.

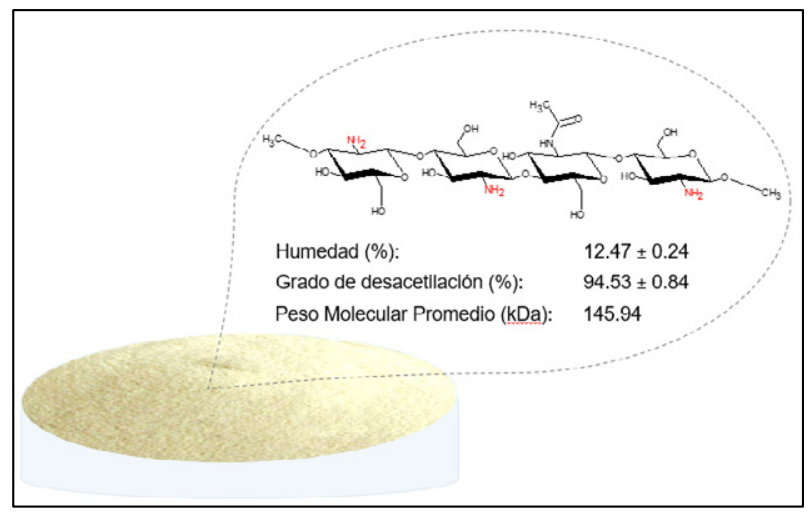

Figura 5. Caracterización del $\beta$-Quitosano.

\section{Cuantificación de la concentración de PDGF-BB in vitro:}

La cantidad de PDGF-BB liberada en el tiempo a partir del hidrogel con $6 \%$ de lisado plaquetario difiere al variar el $\mathrm{pH}$ de 6,5 a 7,4. Tal y como muestra la tabla 1, durante las 32 horas, las concentraciones de PDGF-BB alcanzadas a pH de medio 6,5, fueron superiores a aquellas concentraciones halladas a $\mathrm{pH}$ de medio 7,4. Como se observa en la figura 3 , ambas curvas siguen una tendencia similar; además, transcurrida una hora las concentraciones difieren en un $27 \%$; a las 3 horas, $33 \%$; a las 7 y 10 horas, $31 \%$; a las 20 horas, $29 \%$ y a las 32 horas, $26 \%$. 
Tabla 1. Concentraciones promedio de PDGF-BB $(\mathrm{pg} / \mathrm{mL})$ a partir de hidrogeles con $6 \%$ de lisado plaquetario en medios con $\mathrm{pH} 6,5$ y $\mathrm{pH} 7,4$.

\begin{tabular}{|c|c|c|}
\hline \multirow{2}{*}{ Tiempo (horas) } & \multicolumn{2}{|c|}{ Concentración promedio de PDGF-BB (pg/mL) liberado } \\
\cline { 2 - 3 } & En medio con pH 6,5 & En medio con pH 7,4 \\
\hline 0 & 0,00 & 0,00 \\
1 & 1827,14 & 1321,61 \\
3 & 3852,28 & 2555,97 \\
7 & 5986,64 & 4091,70 \\
10 & 8370,75 & 5780,86 \\
20 & 10604,03 & 7566,17 \\
32 & 12687,66 & 9396,58 \\
\hline
\end{tabular}

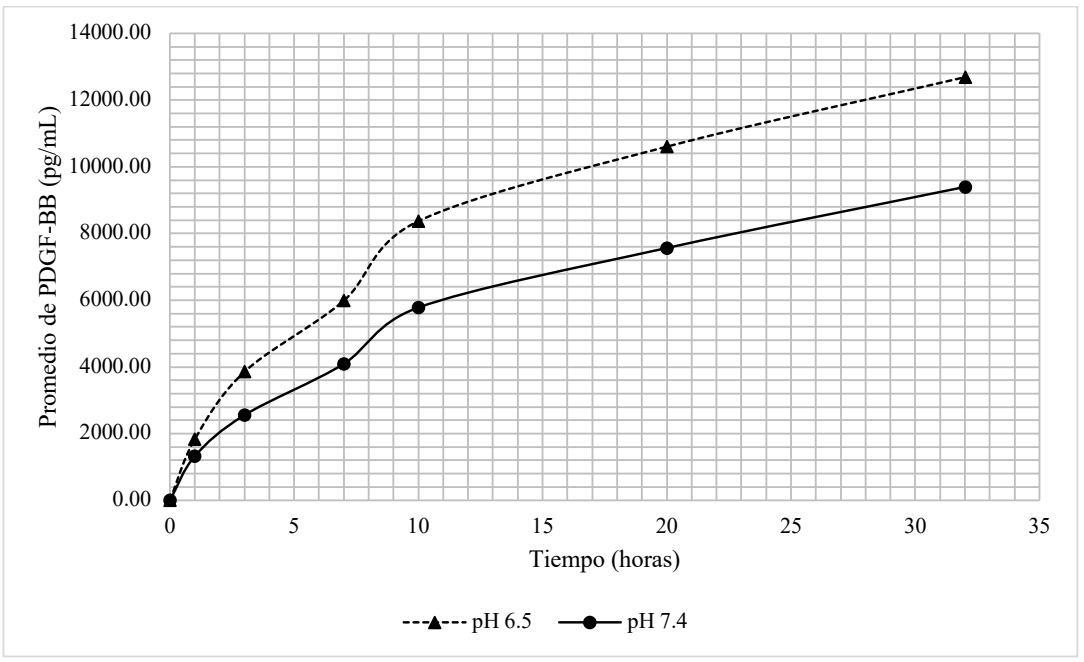

Figura 6. Distribución de las concentraciones promedio de PDGF-BB $(\mathrm{pg} / \mathrm{mL})$ a partir de hidrogeles con $6 \%$ de lisado plaquetario en medios con $\mathrm{pH} 6,5$ y pH 7,4.

Por el contrario, de acuerdo a la tabla 2 y la figura 4, la cantidad de PDGF-BB liberada en el tiempo a partir del hidrogel con $12 \%$ de lisado plaquetario no difiere al variar el $\mathrm{pH}$ de 6,5 a 7,4. 
Tabla 2. Concentraciones promedio de PDGF-BB (pg/mL) a partir de hidrogeles con $12 \%$ de lisado plaquetario en medios con $\mathrm{pH} 6,5$ y $\mathrm{pH} 7,4$.

\begin{tabular}{|c|c|c|}
\hline \multirow{2}{*}{ Tiempo (horas) } & Concentración promedio de PDGF-BB (pg/mL) liberado \\
\cline { 2 - 3 } & En medio con pH 6,5 & En medio con pH 7,4 \\
\hline 0 & 0,00 & 0,00 \\
20 & 2784,36 & 2856,95 \\
32 & 5491,42 & 5387,39 \\
56 & 8123,47 & 8014,33 \\
80 & 10647,11 & 10655,14 \\
104 & 13111,28 & 13079,27 \\
131 & 15503,00 & 15372,99 \\
\hline
\end{tabular}

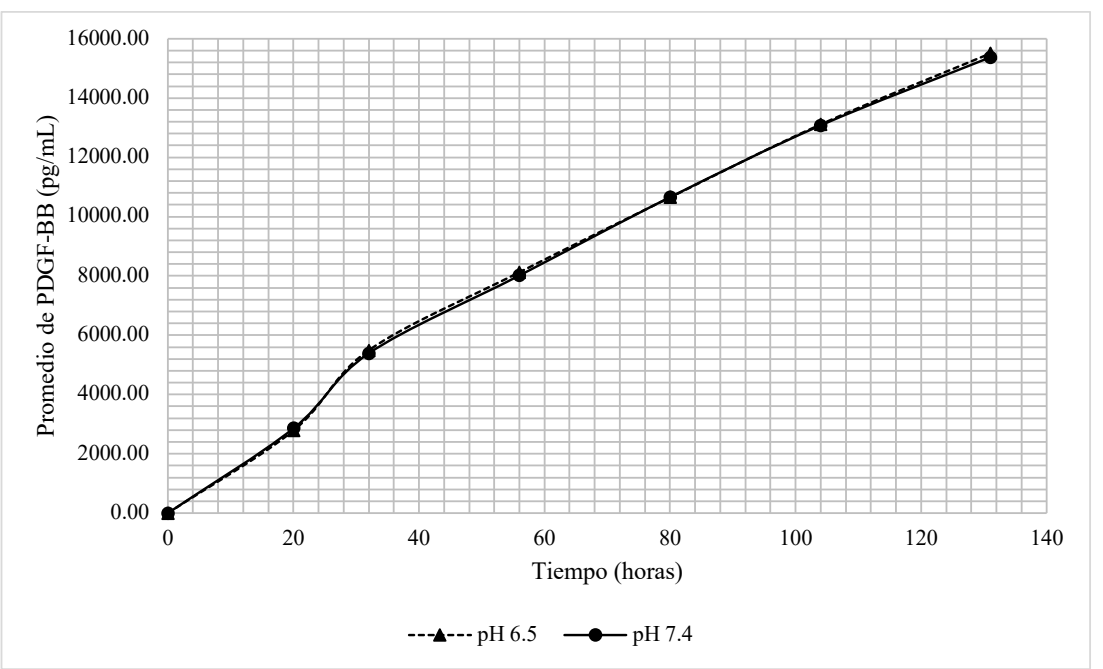

Figura 6. Distribución de las concentraciones promedio de PDGF-BB $(\mathrm{pg} / \mathrm{mL})$ a partir de hidrogeles con $12 \%$ de lisado plaquetario en medios con $\mathrm{pH} 6,5$ y $\mathrm{pH} 7,4$.

\section{Contraste de hipótesis}

Los datos para los dos grupos: hidrogel con $6 \%$ de LP e hidrogel con $12 \%$ de LP, siguen una distribución normal, mas no presentan linealidad ni homogeneidad de varianza. Es así que se usó la prueba de Kruskal-Wallis para contrastar la hipótesis nula en ambos grupos. Los resultados, como muestra la tabla 3, respaldan las figuras 3 y 4 . 
Tabla 3. Contraste de hipótesis con la prueba Kruskal-Wallis.

\begin{tabular}{|l|l|l|l|}
\hline $\begin{array}{c}\text { Hidrogel } \\
\text { con }\end{array}$ & \multicolumn{1}{|c|}{ Hipótesis nula } & $\begin{array}{c}\text { Significancia } \\
\text { (p) }\end{array}$ & \multicolumn{1}{|c|}{ Decisión } \\
\hline $6 \% \mathrm{LP}$ & $\begin{array}{l}\text { La distribución de concentración de } \\
\text { PDGF-BB }(\mathrm{pg} / \mathrm{mL}) \text { es la misma entre } \\
\text { las categorías de } \mathrm{pH} .\end{array}$ & $<0,05$ & $\begin{array}{l}\text { Rechaza la } \\
\text { hipótesis nula }\end{array}$ \\
\hline $12 \% \mathrm{LP}$ & $\begin{array}{l}\text { La distribución de concentración de } \\
\text { PDGF-BB }(\mathrm{pg} / \mathrm{mL}) \text { es la misma entre } \\
\text { las categorías de } \mathrm{pH} .\end{array}$ & $>0,05$ & $\begin{array}{l}\text { Conserva la } \\
\text { hipótesis nula }\end{array}$ \\
\hline Se muestran significancias asintóticas. El nivel de significación es $0,05$. & \\
\hline
\end{tabular}

Trabajos como el de Yang et al, 2011 argumentan que la aplicación de PRP en úlceras inducidas en ratas no tiene actividad cicatrizante; esto debido a la liberación instantánea de factores de crecimiento (FCs) y el número limitado de receptores. Como consecuencia, la fracción de FCs que no llegó a unirse a su receptor, se inactiva, influyendo negativamente en el proceso cicatrización ${ }^{14}$. Es por ello la importancia de usar quitosano como matriz de formas farmacéuticas, y así lograr retardar la liberación ${ }^{12,13}$.

El quitosano no es una entidad química, por lo que es necesario caracterizar la muestra usada en cada investigación. Como se aprecia en la figura 2, las características del $\beta$-quitosano como GD elevado $\left(94,53 \%\right.$ ) y PM bajo $(145,94 \mathrm{kD})^{9,10}$, otorgan al $\beta$-quitosano propiedades particulares como buena solubilidad, mayor participación en interacciones iónicas, actividad antimicrobiana y fungistática; todas ellas basadas en la presencia de mayor cantidad de grupos amina. Estos grupos funcionales son potencialmente protonables, lo que mejora la solubilidad, interacciones iónicas y la actividad fungistática. Por otro lado, la actividad antimicrobiana depende más del PM, el cual mientras menor sea, facilita el movimiento e interacción con la membrana celular del microbio ${ }^{15,16}$.

La forma farmacéutica propuesta hace uso de este $\beta$-quitosano como matriz en el hidrogel para retardar la liberación de PDGF-BB, cuya medición con respecto al tiempo se muestra en las figuras 3 y 4 . La figura 3 muestra que la liberación de PDGF-BB del hidrogel elaborado con $6 \%$ de LP sí está influenciada por el $\mathrm{pH}$. La tendencia de ambas curvas es similar, pues la liberación de PDGF-BB se da de manera muy rápida, conocido como efecto burst. La influencia del $\mathrm{pH}$ en la liberación de PDGF-BB se explica al considerar la composición de aminoácidos y su punto isoeléctrico ( $\mathrm{pH} 9,8)$. Expuestos a un medio ligeramente ácido (pH 6,5), la gran cantidad de aminoácidos básicos y polares estarán en su forma catiónica; algunos de los aminoácidos polares estarán en su forma neutra y/o cargados positivamente; los aminoácidos ácidos estarán en su forma neutra y/o cargado positivamente; mientras que los aminoácidos básicos, estarán cargados positivamente. Todo conlleva a una repulsión de cargas entre PDGF-BB y la matriz de $\beta$-quitosano de manera brusca. 
La figura 4 demuestra que la liberación de PDGF-BB del hidrogel elaborado con $12 \%$ de LP no está influenciada por el pH. En este caso las curvas además de seguir la misma tendencia, llegan a superponerse. Estos resultados no pueden ser explicados solo por interacciones iónicas, pues de ser así se verían influenciados por el $\mathrm{pH}$. Es válido pensar que el aumento en el porcentaje de LP implica un aumento de los grupos reactivos para generar nuevos enlaces covalentes. Pero, no todos estos enlaces son fuertes. La formación de bases de Schiff es otra posible reacción entre los grupos ceto o carboxilo, presente en aminoácidos de PDGF-BB con las aminas secundarias del $\beta$-quitosano para generar enlaces imina. Estos enlaces son fácilmente hidrolizables a $\mathrm{pH}$ ácidos y estables en $\mathrm{pH}$ neutros o alcalinos ${ }^{7,15}$.

En el mismo trabajo de Yang et al, 2011 la liberación sostenida de PDGF-BB a partir de un gel de fibrina-heparina lo atribuye a interacciones electrostáticas entre los FCs y la heparina (macromolécula aniónica) ${ }^{14}$. Esta es la razón por la cual esta forma farmacéutica incluye glutaraldehído en pequeñas concentraciones con la finalidad de asegurar la liberación retardada. Sin embargo, los resultados obtenidos en este trabajo dan motivos para no excluir la formación de enlaces imina ni interacciones iónicas, abriendo la posibilidad de prescindir el uso de glutaraldehído.

La figura 4 consolida el logro de diseñar una forma farmacéutica que retarde la liberación de PDGF-BB usando un hidrogel cuya matriz de $\beta$-quitosano carga $12 \%$ de LP. No obstante, una de las grandes interrogantes es conocer realmente cómo es que PDGF-BB se entrampa a la matriz de $\beta$-quitosano. Los argumentos previos son especulaciones hechas a partir de la teoría, pues se requerían de otros equipos para demostrar la formación de nuevos enlaces.

\section{CONCLUSIONES}

- $\quad$ El $\beta$-quitosano con el que se trabajó, tiene $12,47 \%$ de humedad, un grado de desacetilación de $94,53 \%$ y peso molecular promedio de $145,94 \mathrm{kD}$.

- La liberación del factor de crecimiento derivado de plaquetas-BB del hidrogel de $\beta$-quitosano con $6 \%$ de lisado plaquetario sí está influenciada por el $\mathrm{pH}(\mathrm{p}<0,05)$.

- La liberación del factor de crecimiento derivado de plaquetas-BB del hidrogel de $\beta$-quitosano con $12 \%$ de lisado plaquetario no está influenciada por el $\mathrm{pH}(\mathrm{p}>0,05)$.

\section{AGRADECIMIENTOS}

Proyecto financiado por el Programa Nacional de Innovación para la Competitividad y Productividad (Innóvate Perú), de acuerdo al contrato N 149-PNICP-PIAP-2015. 


\section{REFERENCIAS BIBLIOGRÁFICAS}

1. Schreml S, Szeimies R-M, Karrer S, Heinlin J, Landthaler M, Babilas P. The impact of the $\mathrm{pH}$ value on skin integrity and cutaneous wound healing. J Eur Acad Dermatol. 2010;24(4):373-378.

2. Boateng J, Catanzano O. Advanced Therapeutic Dressings for Effective Wound Healing-A Review. J Pharm Sci. 2015;104(11):3653-3680.

3. Everts PAM, Knape JTA, Weibrich G, Schönberger JPAM, Hoffmann J, Overdevest EP, et al. Platelet-rich plasma and platelet gel: a review. J Extra Corpor Technol. 2006;38(2):174-187.

4. Lynch SE, Nixon JC, Colvin RB, Antoniades HN. Role of platelet-derived growth factor in wound healing: synergistic effects with other growth factors. Proc Natl Acad Sci USA. 1987;84(21):7696-700.

5. Busilacchi A, Gigante A, Mattioli-Belmonte M, Manzotti S, Muzzarelli RAA. Chitosan stabilizes platelet growth factors and modulates stem cell differentiation toward tissue regeneration. Carbohydr Polym. 2013;98(1):665-676.

6. Bhattarai N, Gunn J, Zhang M. Chitosan-based hydrogels for controlled, localized drug delivery. Adv Drug Deliv Rev. 2010;62(1):83-99.

7. Nakamatsu J, Rodríguez N. Química de la Quitina y el Quitosano. En: Quitina y Quitosano: Obtención, caracterización y aplicaciones. $1^{\circ}$ Edición. Lima - Perú: Fondo Editorial de la PUCP; 2004. p. 74-102.

8. Arguelles W, Heras A, Acosta N, Galed G, Gallardo A, Miralles B, Peniche C, San Román J. Caracterización de Quitina y Quitosano. En: Quitina y Quitosano: Obtención, caracterización y aplicaciones. $1^{\circ}$ Edición. Lima - Perú: Fondo Editorial de la PUCP; 2004. p. 155-206.

9. Kasaai MR. Calculation of Mark-Houwink-Sakurada (MHS) equation viscometric constants for chitosan in any solvent-temperature system using experimental reported viscometric constants data. Carbohydr Polym. 2007;68(3):477-488.

10. Rinaudo M, Milas M, Dung PL. Characterization of chitosan. Influence of ionic strength and degree of acetylation on chain expansion. Int J Biol Macromol. 1993;15(5):281-5.

11. Seabaugh KA, Thoresen M, Giguère S. Extracorporeal Shockwave Therapy Increases Growth Factor Release from Equine Platelet-Rich Plasma in vitro. Front Vet Sci. 2017 Dec 7;4:205. doi: 10.3389/fvets.2017.00205.

12. Beppu MM, Vieira RS, Aimoli CG, Santana CC. Crosslinking of chitosan membranes using glutaraldehyde: Effect on ion permeability and water absorption. J Membrane Sci. 2007;301(1-2):126-130. 
13. Kutlu B, Tiğlı Aydın RS, Akman AC, Gümüşderelioglu M, Nohutcu RM. Platelet-rich plasma-loaded chitosan scaffolds: Preparation and growth factor release kinetics. J Biomed Mater Res B Appl Biomater. 2013;101(1):28-35.

14. Yang HS, Shin J, Bhang SH, Shin J-Y, Park J, Im G-I, et al. Enhanced skin wound healing by a sustained release of growth factors contained in platelet-rich plasma. Exp Mol Med. 2011; 43(11): 622-629.

15. Pastor de Abram A, Higuera I. Generalidades. En: Quitina y Quitosano: Obtención, caracterización y aplicaciones. $1^{\circ}$ Edición. Lima - Perú: Fondo Editorial de la PUCP; 2004. p. 25-71.

16. Goy RC, Britto D de, Assis OBG. A review of the antimicrobial activity of chitosan. Polímeros. 2009;19(3):241-247. 